\title{
Terapia Comportamental Infantil: um panorama sobre o uso de estratégias lúdicas
}

\section{Child Behavioral Therapy: an overview of the use of play strategies \\ Terapia Comportamental Infantil: un panorama sobre el uso de estrategias lúdicas}

\author{
Maria Fernanda Monteiro', Mariana Amaral ${ }^{2}$
}

[1] Centro Universitário Filadélfia (UniFil); Universidade Federal do Paraná (UFPR) [2] Centro Universitário Filadélfia (UniFil); Pontifícia Universidade Católica de São Paulo (PUC-SP) I Título abreviado: Estratégias Lúdicas na Terapia Comportamental Infantil I Endereço para correspondência: Rua Prefeito Hugo Cabral 1174, apto.602. Londrina - PR. CEP:86020-111 I Email: monteiro.mfer@yahoo.coml doi: 10.18761/PAC.v10.n2.a4

Resumo: A terapia infantil baseada nos princípios da Análise do Comportamento tem passado por avanços teóricos e conceituais, que a levaram a abranger contextos mais amplos do que apenas o comportamento problema, abordando comportamentos privados e aumentando a participação da criança no processo terapêutico. A utilização de estratégias lúdicas é uma forma de auxiliar a inclusão da criança na terapia, tornando o processo mais divertido e dinâmico, facilitando a obtenção de dados relevantes, entre outros benefícios. A presente pesquisa investigou o uso das estratégias lúdicas por terapeutas comportamentais infantis por meio de um formulário. Participaram da pesquisa vinte e sete psicólogos analistas do comportamento que atuam no contexto infantil. Os resultados indicaram que os participantes utilizam ampla variedade de recursos desde a intervenção até o encerramento da terapia, com objetivos relacionados à expressão emocional, fortalecimento do vínculo terapêutico, entre outros. Tais dados corroboram a literatura clínica no que tange à importância da utilização de recursos lúdicos em sessão e de objetivos bem estabelecidos para tais recursos, pautados na avaliação funcional de cada caso.

Palavras-chave: terapia comportamental infantil; estratégias lúdicas; recursos lúdicos. 
Abstract: Child therapy based on the principles of Behavior Analysis has undergone theoretical and conceptual advances that have led it to encompass broader contexts than just problem behavior, addressing private behaviors and increasing child participation in the therapeutic process. The use of play strategies is a way of helping the child to be included in the therapy, making the process more fun and dynamic, facilitating the obtaining of relevant data, among other benefits. The present research aimed to produce knowledge about the use of play strategies by child behavior therapists through a form. Twenty-seven behavioral psychologists who work with children participated in the study. The results indicated that the participants used a wide variety of resources from intervention to the end of therapy, with objectives related to emotional expression, strengthening of the therapeutic bond, among others. These data corroborate the clinical literature regarding the importance of the use of recreational resources in session and well-established objectives for such resources, based on the functional evaluation of each case.

Keywords: child behavioral therapy; play strategies; recreational resources.

Resumen: La terapia infantil basada en los principios del Análisis del Comportamiento ha pasado por avances teóricos y conceptuales que la llevaron a abarcar contextos más amplios que sólo el comportamiento problemático, abordando comportamientos privados y aumentando la participación del niño en el proceso terapéutico. La utilización de estrategias lúdicas es una forma de auxiliar la inclusión del niño en la terapia, haciendo el proceso más divertido y dinámico, facilitando la obtención de datos relevantes, entre otros beneficios. La presente investigación buscó producir conocimiento acerca del uso de las estrategias lúdicas por terapeutas comportamentales infantiles a través de un formulario. Participaron de la investigación veintisiete psicólogos analistas del comportamiento que actúan en el contexto infantil. Los resultados indicaron que los participantes utilizan amplia variedad de recursos desde la intervención hasta el cierre de la terapia, con objetivos relacionados a la expresión emocional, fortalecimiento del vínculo terapéutico, entre otros. Tales datos corroboran la literatura clínica en lo que se refiere a la importancia de la utilización de recursos lúdicos en sesión y de objetivos bien establecidos para tales recursos, pautados en la evaluación funcional de cada caso.

Palabras clave: terapia comportamental infantil; estrategias lúdicas; recursos lúdicos. 
A terapia infantil baseada nos princípios da Análise do Comportamento possui um histórico de constantes transformações, partindo dos trabalhos e experimentos realizados com base nas obras de Watson sobre eliminação de fobias, passando pelo período de Modificação do Comportamento Infantil, e chegando até à sua configuração atual (Conte \& Regra, 2000; Guerrelhas, Bueno, \& Silvares, 2000; Haber \& Carmo, 2007; Vermes, 2012).

Um dos principais aspectos que caracteriza a Terapia Comportamental Infantil (TCI) atual e a diferencia dos períodos anteriores é a participação ativa da criança no processo terapêutico (Conte \& Regra, 2000; Regra 2000). A presença da criança no setting terapêutico trouxe a necessidade da inclusão de formas de obtenção de dados e intervenções que se adaptassem à realidade do cliente, sendo a prática de brincadeiras e o uso de estratégias lúdicas os procedimentos adotados pelos terapeutas para suprir tais necessidades (Bezerra, Teixeira, \& Palha, 2013; Conte \& Regra, 2000; Del Prette \& Meyer, 2012; Gadelha \& Menezes, 2004; Guerrelhas et al., 2000; Regra, 2000).

O uso de jogos e brincadeiras no contexto terapêutico é uma estratégia amplamente utilizada em diversas abordagens (Aberastury, 1992; Aragão \& Azevedo, 2001; Conte, 1999; Fulgencio, 2008; Oaklander, 1980; Schmidt \& Nunes, 2014). Embora Skinner não tenha abordado o tema de forma extensa, ele propõe definições que diferenciam o jogar e o brincar livre, nas quais o jogar é uma atividade possui regras verbais com contingências de reforçamento planejadas, e o brincar não seria necessariamente controlado pelo ambiente verbal, sendo que ambos possuem fortes funções reforçadoras positivas (Skinner, 1991).

A utilização de jogos e brincadeiras com objetivos específicos TCI é denominada estratégia lúdica, e pode incluir uma grande diversidade de instrumentos, como jogos, pinturas, colagens, música, histórias, metáforas, entre muitos outros (Gadelha $\&$ Menezes, 2004). Entre as vantagens que as estratégias lúdicas podem conferir à TCI, estão: (a) facilitar a construção do vínculo terapêutico, (b) avaliar o grau de desenvolvimento da criança, (c) evocar e intervir em comportamentos ocorridos na própria sessão e (d) modelar respostas mais adap- tativas (Banaco, 1997; Conte \& Regra, 2000; Haber \& Carmo, 2007; Regra, 1997).

A escolha pela aquisição e utilização de determinada estratégia lúdica é muito mais eficaz quando pautada nas informações obtidas pelo terapeuta por meio da avaliação funcional do caso, e tal estratégia pode ser usada como um instrumento para obter dados importantes para essa avaliação (Del Prette \& Meyer, 2012). Conhecer as variáveis que mais influenciam a escolha por certa estratégia lúdica em um caso específico pode auxiliar no processo de tomada de decisão de outros terapeutas que estejam atuando em casos que possuam características semelhantes, seja em relação à queixa ou aos objetivos estabelecidos na sessão (Moura \& Venturelli, 2004).

Embora o uso de estratégias lúdicas esteja presente na literatura da clínica comportamental infantil, a análise das variáveis envolvidas no processo de tomada de decisão a respeito das estratégias lúdicas específicas a serem empregadas em cada caso se restringe à prática clínica, e é descrita apenas em alguns estudos de caso clínico. Segundo Moura e Venturelli (2004), as varáveis que direcionam as escolhas dos terapeutas em suas práticas são pouco conhecidas, e ainda precisam ser estudadas e discutidas.

O presente estudo tem como objetivo geral investigar as variáveis envolvidas no processo de tomada de decisão de psicólogos que atuam utilizando TCI a respeito do uso de estratégias lúdicas, bem como os objetivos estabelecidos para cada atividade lúdica em sessão, por meio de aplicação de um formulário online.

\section{Método}

\section{Participantes}

Participaram da pesquisa vinte e sete psicólogos que atuam na clínica com a população infantil $(\mathrm{H}=6, \mathrm{M}=21)$. A faixa etária dos participantes variou de 22 a 53 anos $(M=29, S D=7,665$.) Dezenove participantes (76\%) formaram-se há menos de cinco anos, caracterizando a amostra com predominância de Psicólogos recém-formados ou com pouco tempo de atuação. 


\section{Instrumentos}

Os instrumentos utilizados para a pesquisa foram dois formulários elaborados pelas autoras. O primeiro consistiu em um Formulário de Avaliação (Figura 1), utilizado na fase inicial da pesquisa, com objetivo de avaliar a clareza e objetividade das questões do formulário principal. O formulário foi composto por cinco questões em escala Likert e contou também com um espaço opcional para respostas abertas em caso de feedbacks específicos para cada questão.
O Formulário Principal (Figura 2) foi composto por treze questões, abrangendo aspectos de identificação e da atividade clínica do terapeuta participante (ex.: cidade e tempo de atuação na TCI), e aspectos relacionados à aquisição, escolha e utilização dos recursos lúdicos em sessão. Ambos os formulários foram compartilhados com os participantes da pesquisa por meio da plataforma "Google Formulários”. Para o envio dos formulários e categorização dos dados foi utilizado um notebook.

Endereço de e-mail:

1. As questões do formulário foram compreendidas com clareza

$\begin{array}{lllllll}\begin{array}{l}\text { Discordo } \\ \text { plenamente }\end{array} & 1 & 2 & 3 & 4 & 5 & \begin{array}{l}\text { Concordo } \\ \text { plenamente }\end{array}\end{array}$

2. As alternativas de resposta apresentadas nas questões condizem com minha experiência clínica

$\begin{array}{lllllll}\begin{array}{l}\text { Discordo } \\ \text { plenamente }\end{array} & 1 & 2 & 3 & 4 & 5 & \begin{array}{l}\text { Concordo } \\ \text { plenamente }\end{array}\end{array}$

Comentários:

3. A quantidade de questőes do formulário é adequada

$\begin{array}{lllllll}\begin{array}{l}\text { Discordo } \\ \text { plenamente }\end{array} & 1 & 2 & 3 & 4 & 5 & \begin{array}{c}\text { Concordo } \\ \text { plenamente }\end{array}\end{array}$

Comentários:

4. As questões do formulário são condizentes com a proposta de avaliar o uso de estratégias lúdicas em sessão

$\begin{array}{lllllll}\begin{array}{l}\text { Discordo } \\ \text { plenamente }\end{array} & 1 & 2 & 3 & 4 & 5 & \begin{array}{l}\text { Concordo } \\ \text { plenamente }\end{array}\end{array}$

Comentários:

5. Você tem alguma sugestão para a melhoria do formulário? Descreva-a abaixo.

Figura 1: Formulário de avaliação 
O objetivo do presente formulário é investigar o uso de estratégias lúdicas e seus objetivos no contexto terapêutico. Por favor, preencha de acordo com sua experiência clínica.

1. Endereço de e-mail

2. Cidade

3. Sexo

4. Idade

5. Em qual Instituição de Ensino você se formou?

6. Em que ano você se formou?

7. Há quanto tempo atua na clínica infantil?

(Apenas uma alternativa pode ser selecionada.)
( ) 0 a 5 anos
( ) 5 a 10 anos
( ) 10 a 15 anos
( ) acima de 15 anos

8. Você atua em:

(Mais de uma alternativa pode ser selecionada. Marque todas que se aplicam.)

( ) Clínica particular

( ) Contexto escolar, realizando atendimento clínico

( ) Instituição filantrópica

( ) Outro:

9. Para qual "momento" da terapia você utiliza recursos lúdicos?

(Mais de uma alternativa pode ser selecionada. Marque todas que se aplicam.)

( ) Contato inicial/Conceituação do caso

( ) Avaliação Funcional

( ) Intervenção

( ) Encerramento

( ) Outro:

10. Assinale o(s) recurso(s) lúdico(s) que utiliza em sessão:

(Mais de uma alternativa pode ser selecionada. Marque todas que se aplicam.)

( ) Atividades manuais (desenho, recortes, pintura, argila, massinha, etc.)

( ) Jogos de tabuleiro (xadrez, Jogo da Vida, Bando Imobiliário, etc.)

( ) Jogos de cartas (Uno, baralho, Conversinha, etc.)

( ) Histórias, fantasias e dramatizações (contação de histórias, uso de bonecos, uso de metáforas, etc.)

( ) jogos eletrônicos (jogos de aplicativos, vídeo games, etc.)

( ) Outro:

11. Selecione os fatores que mais influenciam em sua escolha na aquisição e uso de recursos lúdicos na terapia (Escolha de uma a três alternativas.)

( ) Experiência clínica

( ) Experiência profissional com crianças

( ) Aprendizado na graduação/pós-graduação sobre o tema

( ) Aprendizado em cursos de formação sobre o tema

( ) Indicações da literatura da área de clínica comportamental infantil

( ) Recursos extraídos da internet e/ou outros meios de comunicação

( ) Indicações de colegas

12. Qual seu critério de escolha do recurso lúdico específico a ser usado em sessão?

(Considere o "Critério 1" como o de maior prioridade, e o "Critério 3" como o de menor prioridade. Marque apenas uma alternativa por linha)

\begin{tabular}{|l|l|l|l|}
\cline { 2 - 4 } \multicolumn{1}{c|}{} & Critério 1 & Critério 2 & Critério 3 \\
\hline $\begin{array}{l}\text { De acordo com o comportamento que } \\
\text { será trabalhado em sessão }\end{array}$ & & & \\
\hline $\begin{array}{l}\text { De acordo com o tempo disponível em } \\
\text { sessão }\end{array}$ & & & \\
\hline De acordo com a vontade da criança & & & \\
\hline
\end{tabular}

13. Liste os cinco principais recursos lúdicos que você utiliza em sessão e para quais queixas eles geralmente são utilizados (Ex: Desenho livre - dificuldade em expressar sentimentos).

Figura 2: Formulário principal, após correções apontadas pelo Formulário de avaliação 


\section{Procedimento}

$\mathrm{Na}$ fase inicial da pesquisa, foi realizada a avaliação do formulário. Os avaliadores foram dez terapeutas comportamentais que atuavam como terapeutas infantis ou como docentes de instituições de ensino superior realizando supervisões no contexto clínico infantil. Os mesmos foram convidados a participar desta pesquisa via e-mail, sendo o contato dos participantes obtido por meio da indicação da orientadora da pesquisa. Uma versão piloto do formulário foi enviada via e-mail, juntamente com o Formulário de Avaliação (Figura 1). Os participantes contaram com um prazo de dez dias, a partir do recebimento do e-mail, para responder os formulários e fornecer seus feedbacks à pesquisadora. Dos dez terapeutas selecionados, dois responderam aos formulários no prazo estabelecido, e com base nestas respostas, as pesquisadoras analisaram as avaliações recebidas, fazendo modificações e correções no formulário original de acordo com os comentários dos avaliadores.

Após a avaliação e correção do formulário piloto, a versão final (Formulário Principal - Figura 2) foi enviada via e-mail aos demais participantes da pesquisa, sendo os primeiros participantes excluídos desta fase. Os mesmos contaram com um prazo de vinte dias para o preenchimento, sendo que os formulários preenchidos foram enviados automaticamente à pesquisadora pela plataforma Google Formulários. O conteúdo das respostas às questões fechadas foi sistematizado pela própria plataforma, sendo os resultados analisados pelas pesquisadoras. O conteúdo das respostas às questões abertas foi categorizado e analisado pelas pesquisadoras, sendo as análises apresentadas a seguir.

\section{Resultados e Discussão}

A primeira questão respondida pelos participantes, após o levantamento de dados pessoais, era sobre em qual "momento" da terapia os mesmos utilizavam estratégias lúdicas: "Intervenção", "Contato inicial", "Avaliação funcional" e "Encerramento", sendo que mais de uma categoria poderia ser selecionada. Os dados obtidos indicam que a intervenção é a principal etapa do processo terapêutico no qual estratégias lúdicas são utilizadas, sendo citado pela totalidade dos participantes. As demais etapas também foram expressivamente citadas, sendo que $44 \%$ da amostra (11 participantes) relataram utilizar estratégias lúdicas em todos os "momentos" da terapia.

As categorias "Contato Inicial" e "Avaliação Funcional" foram citadas por $88 \%$ (22 respostas) e $80 \%$ (20 respostas), respectivamente, e a categoria "Encerramento" foi citada por $60 \%$ dos participantes (15 respostas). A menor frequência de estratégias lúdicas relatadas pelos participantes nos "momentos" de Avaliação Funcional e Encerramento sugere a utilização de outras estratégias, como a preferência por interações verbais; pela presença dos pais, especialmente no encerramento do processo terapêutico; ou mesmo pelo uso de materiais que não sejam considerados como lúdicos pelos terapeutas.

Ainda assim, o relato dos participantes a respeito da escolha do uso de estratégias lúdicas em diferentes momentos vai ao encontro da literatura clínica, que caracteriza a utilização de estratégias lúdicas como uma ferramenta útil para o levantamento de dados sobre o cliente, avaliação funcional, intervenção e encerramento (Gadelha \& Menezes, 2004; Moura \& Venturelli, 2004; Regra, 2012; Torres \& Meyer, 2003).

Os participantes foram questionados quanto ao tipo de estratégias lúdicas que utilizam em sessão, podendo selecionar uma ou mais das seguintes categorias: "Atividades manuais"; "Jogos de cartas"; "Jogos de tabuleiro"; "Histórias, fantasias e dramatizações"; "Jogos eletrônicos" e "Outros". Os resultados indicam que os participantes utilizam recursos pertencentes à maioria das categorias, sendo "Atividades manuais" selecionada por 24 participantes (96\%); "Jogos de cartas" e Jogos de tabuleiro", por 23 participantes (92\%); "Histórias, fantasias e dramatizações", citada por 21 participantes (84\%); "Jogos eletrônicos", por nove participantes (36\%); e "Outros", selecionada por seis participantes (24\%).

A citação de variadas categorias pelos participantes é um indicativo da multiplicidade de objetivos e funções que cada recurso pode assumir, e também da variedade de recursos disponíveis a serem utilizados pelos terapeutas. De acordo com Del Prette e Meyer (2012), o tipo de estratégia a ser escolhida para a sessão deve ocorrer em fun- 
ção dos seguintes critérios: objetivos do terapeuta com aquele cliente, o nível de desenvolvimento da criança, e variações das preferências da criança por algum tipo de jogo ou brincadeira. Considerando o caráter único de cada caso, cada estratégia lúdica pode ser utilizada com objetivos diferentes para cada cliente, sendo a avaliação funcional o norteador desta escolha (Vermes, 2012).

$\mathrm{O}$ uso de jogos eletrônicos, como os presentes em videogames ou em aplicativos para dispositivos móveis foram indicados como recursos usados pelos terapeutas em nove respostas (36\%). A frequência reduzida em comparação às outras categorias pode estar relacionada a variáveis como o custo mais elevado dos aparelhos eletrônicos, pouco conhecimento sobre as possibilidades de uso dos jogos, difícil acesso aos aplicativos por conta do idioma, entre outros.

Vale ressaltar, porém, que a utilização de elementos de design dos jogos eletrônicos, descrita como gamificação, tem sido discutida e aplicada por analistas do comportamento em diversos contextos, com resultados que incentivam seu uso (Baranowski, Buday, Thompson, \& Baranowski, 2008; Brown et al., 2016; Morford, Witts, Killingsworth, \& Alavosius, 2014). Desse modo, é possível hipotetizar um aumento do uso dessa estratégia lúdica nos próximos anos.

Como fatores de influência na utilização de estratégias lúdicas, as categorias "Experiência clínica", "Aprendizado na graduação/pós-graduação sobre o tema" e "Aprendizado em cursos de formação sobre o tema" foram as mais citadas pelos participantes. A maior frequência de respostas associada à experiência clínica e no aprendizado em cursos na área indicam que a escolha pela aquisição e utilização de determinados recursos lúdicos advém tanto de experiências "vivenciais" (como a experiência com determinados jogos) quanto "formais" (indicadas em aulas e cursos).

A presença de critérios "formais" na escolha pela aquisição e utilização de recursos pelos terapeutas pode ser considerada vantajosa por indicar a relevância de conhecimentos empíricos divulgados por pares na comunidade científica na prática clínica. Considerando o modelo de prática baseada em evidências, a prática clínica fundamentada em pesquisas auxilia o terapeuta em sua tomada de decisões, integrando o conhecimento científico dos processos psicológicos à experiência clínica (Leonardi \& Meyer, 2015; Reis \& Barbosa, 2018).

Os participantes também foram questionados sobre os critérios para a utilização de determinado recurso ou atividade na sessão. Vinte e três participantes (92\%) definiram o comportamento a ser trabalhado em sessão como primeiro critério para escolher um jogo ou brinquedo. As alternativas "De acordo com o tempo disponível em sessão" e "De acordo com a vontade da criança" se igualaram como segundo e terceiro critério de escolha, com 12 respostas $(48 \%)$.

Considerar o comportamento a ser trabalhado em sessão é um importante critério de escolha, pois envolve a análise dos comportamentos problema $\mathrm{e}$ de como a estratégia lúdica pode abordá-lo. Ainda assim os demais critérios também devem ser considerados. Conte e Regra (2000, p. 105) afirmam, sobre o uso de estratégias lúdicas, que a "agradabilidade não deve ser desvinculada dos objetivos da terapia". Assim, os critérios "de acordo com o tempo disponível em sessão" e "de acordo com a vontade da criança" também pode possuir objetivos muito importantes, como o de identificar possíveis reforçadores, auxiliar na intervenção de comportamentos relacionados à assertividade e tomada de decisões, e de se fortalecer o vínculo terapêutico e a adesão da criança às sessões (Del Prette \& Meyer, 2012; Gadelha \& Menezes, 2004; Moura \& Venturelli, 2004; Vermes, 2012).

Na última questão do formulário, os participantes da pesquisa foram orientados a listar os cinco recursos lúdicos que mais utilizam em sessão, bem como os objetivos para os quais eles geralmente são utilizados. Foram obtidas 125 respostas, as quais foram organizadas de acordo com as seguintes categorias: "Desenhos", "Outras atividades manuais", "Jogos estruturados", "Jogos de cartas", "Histórias, fantasias e dramatizações", "Jogos eletrônicos", e "Outros", sendo que em algumas respostas, foram citados mais de um jogo ou mais de um objetivo. A frequência da citação de cada categoria pode ser observada na Figura 3. 




Figura 3: Porcentagem de ocorrência de cada resposta citando as categorias de recursos lúdicos na questão 13

Observa-se que a frequência de citações que cada categoria obteve confirma a ampla presença de todas as estratégias, com exceção de "Jogos Eletrônicos" e "Outros". Ressalta-se que as categorias "Desenhos" e "Atividades manuais" formaram uma única categoria na questão presente no formulário, porém, foram separadas na categorização de respostas abertas por conta da diferença nos objetivos relatados para cada atividade. Os resultados específicos de cada categoria seguem descritos abaixo.

\section{Desenhos}

O uso de desenhos foi mencionado em 21 respostas (17\%) e descrito como "livre" ou "orientado". Os objetivos mais citados nas respostas foram: "Identificação de expressão de sentimentos" (7 respostas) e "Discussão de temas relacionados à queixa" (4 respostas). Segundo um dos participantes da pesquisa, a atividade de desenhar é empregada "[...] para trazer à tona a discussão sobre vários temas, $e$ para trabalhar diversas queixas, como por exemplo, questões de relacionamento interpessoal, comportamentos inadequados dos mais diversos, etc.". Outro participante descreveu que emprega o recurso para desenvolver a habilidade de "[...] reconhecer e nomear sentimentos".

Também de acordo com a literatura as atividades com desenho têm sido sendo empregadas como forma de se obter informações relevantes sobre a criança, discutir temas difíceis associados a situações específicas, avaliar e treinar habilidades motoras e promover autoconhecimento (Conte, 1997; Conte \& Regra, 2000; Del Prette \& Meyer, 2012; Moraes \& Murari, 2000).

\section{Outras atividades manuais}

Além do desenho, outras atividades manuais foram citadas pelos participantes, como recorte e colagem, confecção de livros e brinquedos, e uso de materiais como blocos e brinquedos para montar, massinha de modelar, bolinhas de gude, tinta 
guache, letras de lixa, pregadores coloridos, entre outros. Esta categoria foi citada 15 vezes, representando $12 \%$ das respostas. O principal objetivo atribuído a essa categoria foi "Treino da coordenação motora e estimulação sensorial” (7 respostas). Um dos participantes descreveu o uso como uma forma de trabalhar "[...] restrição sensorial, habilidade manual, coordenação motora, capacidade de abstração e reconstrução de imagens (tornar concreto o que é pensado)".

A promoção de estimulação sensorial é citada também pela literatura clínica como um dos ganhos possibilitados por atividades manuais, permitindo que a criança entre em contato com seu próprio corpo e sensações físicas ao manusear materiais como argila e massa de modelar, por exemplo (Conte, 1997; Moura \& Venturelli, 2004; Oaklander, 1980).

\section{Jogos estruturados}

Esta categoria, citada em 49 respostas (39\%), inclui jogos de tabuleiro, jogos de cartas e demais jogos que possuam regras estruturadas. Os principais objetivos mencionados para essa categoria foram "Intervenção sobre competitividade e resistência à frustração" (10 respostas), "Seguimento de regras" (9 respostas), "Avaliação e intervenção para repertórios específicos" (9 respostas), "Identificação e expressão de sentimentos" (7 respostas) e "Estimular repertórios de atenção, concentração e memória" (6 respostas).

Os objetivos para o uso de jogos citados pelos participantes vão ao encontro dos objetivos e ganhos descritos pela literatura, como ensinar a lidar com perdas; desenvolver habilidades acadêmicas; desenvolver de repertório de atenção e organização; observar e modelar o repertório de cumprimento de regras; promoção do vínculo terapêutico e colocar a criança em contato com as consequências de seu comportamento (Conte \& Regra, 2000; Del Prette \& Meyer, 2012; Del Rey, 2012; Gadelha \& Menezes, 2004; Tintori, Bast, \& Pitta, 2010; Vermes, 2012).

O seguimento de regras pode ser observado e modelado por meio de situações propostas pelo jogo ou mesmo combinadas entre criança e terapeuta, como seguir as instruções sobre o jogo, aguardar sua vez para jogar e atender a solicita- ções propostas pelo terapeuta (Tintori et al., 2010; Vermes, 2012). O uso de jogos relacionados a conteúdos acadêmicos também é apontado como uma forma de modificar o autoconceito da criança a respeito de sua capacidade em compreender aquele tema, abordando os conteúdos de forma prazerosa e não-punitiva (Conte, 1997; Del Prette \& Meyer, 2012; Tintori et al., 2010).

Sobre repertórios específicos sobre os quais o uso de jogos em sessão pode auxiliar na intervenção, um dos participantes citou "[...] falta de habilidades sociais, repertório de perder e ganhar inadequado, dificuldade de seguimento de regras, transtorno opositor, dificuldade de não seguir regras". Além destas, os jogos estruturados podem auxiliar no manejo de diversos outros tipos de repertórios comportamentais.

Um dos participantes descreve o uso de um baralho que descreve diferentes emoções como forma de evocar temas importantes para a criança: "Baralho de emoções - expressão e identificação de sentimentos, auxílio no relato de situações/experiências vivenciadas e autoconhecimento".

\section{Histórias, fantasias e dramatizações}

Esta categoria foi citada em 31 respostas (25\%) e abrange respostas relacionadas a teatro, role play, uso de livros infantis, bonecos e fantoches, construção de histórias em quadrinhos, entre outros. $\mathrm{O}$ objetivo mais mencionado para essa categoria foi "Avaliação e intervenção para repertórios específicos" (11 respostas). A avaliação e a intervenção com o uso dessa estratégia foi descrita por um dos participantes como: "Faz de conta com fantoches/ objetos - para conceituação do caso, para evocar comportamentos alvo a serem trabalhados em sessão; para trabalhar assertividade, comunicação". Outro participante descreveu: "Contar história baseado em livros infantis que possibilite a criança ver imagens, em um caso específico usei o livro que conta a história de um garotinho que foi para o hospital com medo, mas que fez amigos no local e brincou... Tentei relacionar com o atual momento que o paciente estava passando (uma série de internações cirúrgicas), afim de tentar prepará-lo para o novo ambiente / tornar menos aversivo".

Em relação à intervenção, regras e contingências descritas em histórias infantis auxiliam o terapeuta 
a abordar, direta ou indiretamente, as consequências de seu comportamento (Moura \& Venturelli, 2003; Regra, 2000), e como forma de controle verbal, "são meios importantes de instalar e/ou alterar comportamentos em crianças, ampliando seu repertório, além de sua experiência pessoal, através do contato com as situações vivenciadas pelos personagens de um livro" (Carvalló, 2011, p. 47).

A utilização de exemplos de personagens de histórias de livros, ou mesmo de histórias criadas pela criança, permite que ela fale se si mesma e faça uma análise de seu próprio comportamento de uma forma menos aversiva para ela, além de evocar comportamentos e sentimentos relevantes (Bueno \& Silvares, 2000; Conte, 1997; Del Rey, 2012; Gadelha \& Menezes, 2004; Guerrelhas \& Marinho, 2000; Regra, 2000).

Moraes e Murari (2000) e Moura e Venturelli (2003) citam o role-play como uma forma de treinar a criança a desenvolver novos repertórios de interação social, podendo o terapeuta assumir o papel da criança na dramatização como forma de fornecer a ela modelos de comportamento. Tal prática em sessão permite que a criança entre em contato com contingências presentes em ambientes específicos como a escola, sem a possibilidade de punições da parte de colegas ou professores, por exemplo.

\section{Jogos eletrônicos}

A categoria, presente em cinco respostas (4\%), abrangeu aplicativos, jogos em geral e vídeos educativos. Os objetivos mencionados foram "Desenvolvimento de habilidades sociais" (2 respostas), "Identificação de cores", "Descrição de eventos", "Matching" e "Autonomia" (1 resposta cada).

Apesar do número reduzido de menções, o uso de jogos eletrônicos com fins terapêuticos tem sido utilizado de forma crescente em pesquisas de diversas áreas da psicologia, como desenvolvimento de comportamentos voltados ao cuidado com a saúde, com o meio ambiente, ensino de habilidades acadêmicas, entre outros (Morford et al., 2014).

A introdução do uso de jogos eletrônicos no contexto clínico também pode assumir diversas formas e diversos objetivos, como o uso de softwares voltados para o ensino de habilidades acadêmicas (Iégas \& Haydu, 2015), uso de realidade virtual para tratamentos de fobias (Bouchard, 2014), e di- versos tipos de jogos online e off-line para auxiliar o tratamento de transtornos como autismo e hiperatividade (Wilkinson, Ang, \& Goh, 2008).

\section{Outros}

Estratégias com características diferentes das descritas nas categorias acima também foram citadas, sendo as quatro respostas da categoria (que representaram 3\% do total de respostas): (a) "Comportamento verbal diante para modificação de estratégias comportamentais."; (b) "Conversas informais, sobre temas de interesse relacionando com regras e valores. Para questionar regras e valores que podem influenciar em outros comportamentos problemas (machismo, homofobia, necessidade de ser forte sempre...)."; (c) "Semáforo da raiva: quando as crianças apresentam dificuldades para lidar com o sentimento de Raiva. O vermelho representa as situações que deixam a criança com raiva, o amarelo as estratégias mais adequadas para lidar com a raiva (e a criança deve escolher um) e o verde para seguir."; (d) "Atividade de resolução de problemas, a fim de treinar soluções mais adequadas de problemas, refletindo com a criança as consequências dos comportamentos.".

Embora as atividades descritas acima não necessariamente envolvam brincadeiras e jogos, podem ser consideradas cunhas comportamentais, expondo a criança a novas contingências e possibilitando a aquisição de novos comportamentos (De Rose \& Gil, 2003). Estas estratégias também podem ser acompanhadas de estratégias lúdicas como desenhos ou histórias, como exemplificado por Regra (2000) ao descrever a utilização de relatos verbais e relatos-estória acompanhando a produção de desenhos.

\section{Conclusão}

A presente pesquisa teve como objetivo fornecer um panorama da utilização de recursos lúdicos por terapeutas comportamentais, reunindo informações que possam auxiliar no processo de tomada de decisão sobre qual recurso utilizar diante de casos e queixas específicas. Os resultados obtidos indicaram quais recursos têm sido utilizados pelos terapeutas participantes e com quais objetivos, o que pode indicar estratégias úteis para orientar a aquisição e uso de estratégias lúdicas. É importan- 
te ressaltar que não se pretende, neste manuscrito, normatizar ou limitar as possibilidades de utilização de cada recurso.

A pesquisa teve algumas limitações no que tange à coleta de dados. Primeiramente, por conta do número de participantes (21), não se pode generalizar os dados obtidos a toda a população de psicólogos analistas do comportamento que atuam na clínica infantil. Assim, os dados apresentados devem ser analisados considerando o tamanho e perfil da amostra. Em segundo lugar, embora um questionário eletrônico possibilite um maior alcance geográfico e confira maior praticidade à coleta de dados, o contato das pesquisadoras com os participantes se tornou mais restrito, dificultando o fornecimento de instruções mais detalhadas quando ao preenchimento do formulário e também o acesso a feedbacks e possíveis dúvidas dos participantes, apesar da disposição para contato via e-mail. Outra limitação importante foi o pouco retorno ao formulário de avaliação da pesquisa, o que tornou o número de feedbacks sobre o formulário principal reduzido.

Os dados obtidos pela pesquisa indicaram a utilização de uma ampla variedade de recursos, com diferentes objetivos, o que enriqueceu o conteúdo das respostas e corroborou diversos conceitos apontados pela literatura clínica, como a importância de objetivos específicos para a utilização de cada recurso lúdico, da avaliação funcional como norteador do processo de tomada de decisão, e do grande potencial de tais recursos em todos os momentos da terapia. Observou-se também a utilização de variados tipos de recursos, tanto produzidos especificamente para intervenções clínicas, quanto jogos comerciais e também materiais confeccionados pelos próprios terapeutas e pelas crianças.

Um ponto importante a ser destacado na pesquisa é o uso de recursos eletrônicos, que, embora citado de forma reduzida em comparação aos demais, se fez presente na fala dos participantes. Acredita-se que o uso de tais recursos pode aumentar ao longo dos anos, considerando o avanço das pesquisas na área e diversidade de materiais e objetivos. O levantamento do uso específico de tais estratégias entre psicólogos analistas do comportamento pode ser um objetivo para pesquisas futuras.

Ressalta-se a importância da utilização de estratégias lúdicas em sessão como forma de tornar o processo terapêutico mais eficaz e reforçador para a criança, e da sistematização deste uso como forma de auxiliar o processo de tomada de decisão de outros terapeutas, como afirmam Moura e Venturelli (2004, p. 18): "Refletir sobre o processo clínico de tomada de decisão pode trazer luz às questões que se colocam no dia-a-dia do terapeuta que trabalha com crianças".

\section{Referências}

Aberastury, A. (1992). A criança e seus jogos. Porto Alegre: Artmed.

Aragão, R. M., \& Azevedo, M. R. Z. S. (2001). O brincar no hospital: análise de estratégias e recursos lúdicos utilizados com crianças. Estudos de Psicologia, 18(3), 33-42. https://doi. org/10.1590/S0103-166X2001000200006.

Banaco, R. A. (1997). Fantasia como instrumento de diagnóstico e tratamento: a visão de um behaviorista radical. In Delitti, M. (Org.) Sobre Comportamento e Cognição: A prática da análise do comportamento e da terapia cognitivo-comportamental (Vol. 2, pp. 115-119). São Paulo: Arbytes.

Baranowski, T., Buday, R., Thompson, D. I., \& Baranowski, J. (2008). Playing for Real: Video Games and Stories for Health-Related Behavior Change. Am J Prev Med., 34(1), 74-82. https:// doi.org/10.1016/j.amepre.2007.09.027.

Bezerra, T., Junior, R., Palha, D. (2013). A produção de regras através de brincadeiras em um atendimento clínico comportamental infantil. Perspectivas Em Análise Do Comportamento, 4(2), 70-91. Recuperado de https://www.revistaperspectivas.org/perspectivas/article/view/111

Bouchard, S. (2014). Could virtual reality be effective in treating children with phobias? Expert Review of Neurotherapeutics, 11(2), 207-213. https://doi.org/10.1586/ern.10.196

Brown, M., O’Neill, N., van Woerden, H., Eslambolchilar, P., Jones, M., \& John, A. (2016). Gamification and Adherence to Web-Based Mental Health Interventions: A Systematic Review. JMIR Mental Health, 3(3), e39. https:// doi.org/10.2196/mental.5710 
Carvalló, B. N. (2011). Efeitos de regras descritivas, presentes em histórias infantis, e de monitoramento sobre o comportamento de ler. (Dissertação de Mestrado). Universidade Federal do Pará, Belém, PA, Brasil. Recuperado de http://ppgtpc.propesp.ufpa.br/ARQUIVOS/ dissertacoes/bruna\%20carvallo\%202011.pdf

Conte, F. C. S. (1997). A criança em seu processo terapêutico: reflexões a partir de um estudo de caso. In Delitti, M. (Org.) Sobre Comportamento e Cognição: A prática da análise do comportamento e da terapia cognitivo-comportamental (Vol. 2, pp. 147-154). São Paulo: Arbytes.

Conte, F. C. S. (1999). A terapia de aceitação e compromisso e a criança: uma exploração com o uso de fantasia a partir do trabalho com argila. In Kerbauy, R., \& Wielenska, R. C. (Orgs.), Sobre Comportamento e Cognição: Psicologia comportamental e cognitiva: da reflexão teórica à diversidade da aplicação (Vol. 4, pp. 121-132). Santo André: Esetec.

Conte, F. C. S., \& Regra, J. (2000). A psicoterapia comportamental infantil: novos aspectos. In Silvares, E. F. M. (Org.). Estudos de caso em psicologia clínica comportamental infantil (Vol. 1, pp. 79-136). São Paulo: Papirus.

Del Prette, G., \& Meyer, S. B. (2012). O brincar como ferramenta de avaliação e intervenção na clínica analítico-comportamental infantil. In Borges, N. B., \& Cassas, F. A. (Orgs.). Clínica analítico-comportamental: aspectos teóricos e práticos (pp. 239-250). Porto Alegre: Artmed.

Del Rey, D. (2012). O uso dos recursos lúdicos na avaliação funcional em clínica analíticocomportamental infantil. Em: Borges, N. B., \& Cassas, F. A. (Orgs.). Clínica analítico-comportamental: aspectos teóricos e práticos (pp. 233238). Porto Alegre: Artmed.

De Rose, J. C. C., \& Gil, M. E. C. D. A. (2003). Para uma análise do brincar e de sua função educacional. In Brandão, M. Z. (Org.). Sobre Comportamento e Cognição: a história e os avanços, e a seleção por consequências em ação (Vol. 11, pp.373-382). Santo André: ESETec.

Fulgencio, L. (2008). O brincar como modelo do método de tratamento psicanalítico. Revista Brasileira de Psicanálise, 42(1), 124-136. Recuperado de http://pepsic.bvsalud.org/scielo.
php?script=sci_arttext\&pid=S0486-641X20080 00100013\&lng=pt\&nrm=iso\&tlng $=$ pt

Gadelha, Y. A., \& Menezes, I. N. (2004). Estratégias lúdicas na relação terapêutica com crianças na terapia comportamental. Universitas Ciências da Saúde, 2(1), 57-68. https://doi.org/10.5102/ UCS.V2I1.523

Guerrelhas, F., Bueno, M., Silvares, E. F. D. M. (2000). Grupo de ludoterapia comportamental $\mathrm{x}$ grupo de espera recreativo infantil. Revista Brasileira de Terapia Comportamental e Cognitiva, 2(2), 157-169. Recuperado de http:// www.usp.br/rbtcc/index.php/RBTCC/article/ view/298/238

Haber, G. M., \& Carmo, J. dos S. (2007). O fantasiar como recurso na clínica comportamental infantil. Revista Brasileira de Terapia Comportamental e Cognitiva, IX(1), 45-61. Recuperado de http://www.usp.br/rbtcc/index. $\mathrm{php} / \mathrm{RBTCC} /$ article/view/145/128

Iégas, A. L. de F., \& Haydu, V. B. (2015). Resolução de problemas aritméticos: efeitos de ensino com uma balança virtual. Temas Em Psicologia, 23(1), 83-96. https://doi.org/10.9788/ TP2015.1-06

Marinho, M. L. (2000). A intervenção clínica comportamental com famílias. In Silvares, E. F. M. (Org.). Estudos de caso em psicologia clínica comportamental infantil (Vol. 1, pp. 139-174). São Paulo: Papirus.

Moraes, C. G. D. A., \& Murari, S. C. (2000). A intervenção clínica em grupo de crianças filhas de pais separados. In Silvares, E. F. M. (Org.). Estudos de caso em psicologia clínica comportamental infantil (Vol. 1, pp. 175-198). São Paulo: Papirus.

Leonardi, J. L., \& Meyer, S. B. (2015). Prática baseada em evidências em Psicologia e a história da busca pelas provas empíricas da eficácia das psicoterapias. Psicologia: Ciência e Profissão, 35(4), 1139-1156. https://doi.org/10.1590/19823703001552014

Moura, C. B., \& Venturelli, M. B. (2004). Direcionamentos para a condução do processo terapêutico comportamental com crianças. Revista Brasileira de Terapia Comportamental e Cognitiva, 6(1), 17-30. Recuperado de http://www.usp.br/rbtcc/ index.php/RBTCC/article/view/62/51 
Oaklander, V. (1980) Descobrindo crianças: a abordagem gestáltica com crianças e adolescentes (10a ed.). São Paulo: Summus.

Regra, J. A. G. (1997). Fantasia: instrumento de diagnóstico e tratamento. In Delitti, M. (Org.), Sobre Comportamento e Cognição: A prática da análise do comportamento e da terapia cognitivo-comportamental (Vol. 2, pp. 107-114). São Paulo: Arbytes.

Regra, J. A. G. (2000). Formas de trabalho na psicoterapia infantil: mudanças ocorridas e novas direções. Revista Brasileira de Terapia Comportamental e Cognitiva, 2(1), 79-101. Recuperado de http://www.usp.br/rbtcc/index. php/RBTCC/article/view/291/231

Reis, G. D. A., \& Barbosa, A. J. G. (2018). Formação de terapeutas cognitivo- comportamentais : Um estudo sobre o estado da arte. Revista Brasileira de Terapia Comportamental e Cognitiva, XX(1), 72-85. Recuperado de http://www.usp.br/rbtcc/ index.php/RBTCC/article/view/1138/555

Schmidt, M. B., \& Nunes, M. L. T. (2014). O brincar como método na prática psicanalítica: uma revisão teórica. Revista de Psicologia da IMED, 6(1), 18-24. https://doi.org/10.18256/21755027/psico-imed.v6n1p18-24 .

Silvares, E. F. M. (2000). Terapia comportamental com famílias de crianças agressivas: por que, como e quando. Paideia, 10(19), 24-32. Recuperado de http://www.scielo.br/pdf/paideia/v10n19/04.pdf

Skinner, B. F. (1978). Ciência e comportamento humano. São Paulo: Martins Fontes.

Skinner, B. F. (1991). Questões recentes na análise comportamental. Campinas: Papirus.

Tintori, F., Bast, D. F., Pitta, M. R. (2011) Jogo na terapia comportamental em grupo de crianças com TDAH. Acta Comportamentalia, 19(2), 225-239. Recuperado de http://www. revistas.unam.mx/index.php/acom/article/ view/28029/25904

Torres, I. M., \& Meyer, S. B. (2003). O brinquedo como instrumento auxiliar para a análise funcional em terapia comportamental infantil. Interação em Psicologia, 7(1), 55-63. https://doi. org/10.5380/psi.v7i1
Vermes, J. S. (2012). Clínica comportamental-infantil: a estrutura. In Borges, N. B.; Cassas, F. A. (Orgs.). Clínica analítico-comportamental: aspectos teóricos e práticos (pp. 214-222). Porto Alegre: Artmed.

Wilkinson, N., Ang, R. P., \& Goh, D. H. (2008). Online Video Game Therapy for Mental Health Concerns: A Review. International Journal of Social Psychiatry, 54(4), 370-382. https://doi. org/10.1177/0020764008091659

\section{Informações do Artigo}

Histórico do artigo: submetido em: 07/08/2018

Primeira decisão editorial: 14/12/2019

Aceito em: 17/12/2019 